Sección uno: Ensayo

Mujer y Educación. Un largo camino hacia la igualdad

\title{
Feminismo y educación en los países occidentales desde la Ilustración a la actualidad. Educación, diferencia y desigualdad. ${ }^{1}$
}

Feminism and education in Western countries from the Enlightenment to now. Education, difference and inequality

\author{
Lola Frutos Balibrea \\ Profesora Emérita de Sociología \\ Universidad de Murcia \\ lfb@um.es
}

\section{Resumen}

En el artículo analizo la relación entre Feminismo y Educación en las sociedades desiguales occidentales actuales. Parto del problema de legitimación que tienen todas las sociedades no igualitarias y es cómo conseguir que las personas peor situadas acepten su situación y no se rebelen.

La teoría que aplico se basa en las perspectivas críticas del Feminismo y del Marxismo. Desde este marco teórico, analizo cómo actúa la educación con relación a la desigualdad social en sus distintas dimensiones (clase, género, edad, etnia). En este trabajo incido en la desigualdad de género por ser una de las más ocultas y, por ello, es más fácil su reproducción social. El proceso de legitimación se gesta tempranamente en la socialización familiar, luego en la escuela, con el grupo de iguales, posteriormente en el trabajo, a través de los medios de comunicación, en realidad, durante toda la vida.

La educación, desde la Ilustración, se basa, al menos en teoría, en el mérito, el talento y en la 'igualdad de oportunidades', aunque deberíamos hablar en ocasiones de 'desigualdad de oportunidades'. La desigualdad de género es cada vez más sutil y difícil de investigar, pero hay que sacarla a la luz pues de lo contrario, puede pasar desapercibida.

Palabras clave: Feminismo, Educación, Diferencia, Desigualdad, Socialización, Cambio Social.

${ }^{1}$ Recibido: 10/03/2021 Evaluado: 23/03/2021 Aceptado: 01/04/2021 


\section{Abstract}

In this article I analyze the relationship between Feminism and Education in today's unequal Western societies. I start from the problem of legitimization that all non-egalitarian societies have, that is, how to get the most disadvantaged people to accept their situation and not rebel.

The theory I apply is based on the critical perspectives of Feminism and Marxism. From this theoretical framework I analyze how education acts in relation to social inequality in its different dimensions (class, gender, age, ethnicity). In this work, I focus on gender inequality because it is one of the most hidden and, therefore, its social reproduction is easier. The process of legitimization is gestated early in family socialization, then at school, within the peer group, later at work, through the media, actually, throughout life-

Education, since the Enlightenment, is based, at least in theory, on merit, talent and 'equality of opportunities', although, we should sometimes speak of 'inequality of opportunities'. Gender inequality is increasingly subtle and difficult to investigate, but it must be brought to light, otherwise it may go unnoticed.

Keywords: Feminism, Education, Difference, Inequality, Socialization, Social Change.

\section{Introducción:}

\section{Evolución histórica de la Teoría Feminista y su influencia en la Educación.}

En Europa, tras las guerras de religión se va a producir una profunda reflexividad que emana de la misma sociedad, que sienta las bases de otro orden social basado no en la religión, sino en la ciencia. Se trata de la Ilustración, de la creación de la Democracia, del Siglo de las Luces, de la Modernidad. Surge en Occidente únicamente, pues hubiera sido imposible que se diera en otro tipo de sociedad cerrada; ni en las sociedades esclavistas antiguas, ni en las feudales, ni en sociedades actuales asiáticas en las que predomina la fe, la tradición y no están separados los espacios concernientes a la economía, la moral, la religión, la cultura, la política.

A partir de este momento la legitimación para ocupar una posición en la sociedad procederá del mérito, no del nacimiento como ocurría en las sociedades estamentales del Antiguo Régimen en las que por ley se pertenecía, bien a un estamento dominador y privilegiado como era el Primer Estado (nobleza/señor/altas jerarquías eclesiásticas), o bien, a un estamento dominado, como el Tercer Estado (siervos) y no se daba movilidad social.

La Revolución Francesa constituye un ejemplo de revolución burguesa, aunque se presentaba en nombre del Tercer Estado. Esta revolución supuso una transformación desde una sociedad estamental a una de clases. En la nueva sociedad de clases sí hay movilidad social, siendo el criterio de pertenencia el factor económico, no jurídico, como en la sociedad estamental: quienes eran dueños los medios de producción pertenecerían a una posición alta (burguesía) y quienes sólo contaban con su fuerza de trabajo, constituirían el proletariado. La burguesía que había adquirido formación y escalado posiciones, pero sin derechos políticos -al estar 
encuadrada en el Tercer Estado,- se inspira en los intelectuales de la Ilustración como teoría política revolucionaria.

En efecto, el nuevo orden social llamado Democracia se apoya en los textos ilustrados para generar conceptos nuevos como 'soberanía nacional' frente al origen divino del poder, separación de poderes, derecho a elegir y ser elegido, derecho a la educación.

Los intelectuales ilustrados deslegitiman las bases de la sociedad estamental (Locke, Montesquieu, Voltaire, Rousseau). El Feminismo tiene la misma raíz, aunque es anterior a la Ilustración se deriva de ella. Surge desde el primer momento como conflicto, como crítica a la propia Ilustración, ya que las mujeres son excluidas del nuevo orden social, por lo que históricamente fue una oportunidad perdida. Es decir, cuando los hombres son considerados individuos y ciudadanos, las mujeres son todas idénticas por el hecho de ser mujeres. Ni pueden estudiar, ni elegir y ser elegidas (sufragio), ni tienen derecho a la propiedad.

El sistema de género no es natural sino construido a lo largo de la historia. Y todo sistema social tiene definido lo masculino y lo femenino. Con la Modernidad se establecen dos espacios: uno privado para las mujeres y otro público para los hombres cuya valoración es muy distinta. Los rasgos de cada espacio están resumidos en la siguiente tabla.

\begin{tabular}{|c|c|}
\hline \multicolumn{2}{|c|}{ DIVISIÓN DEL ESPACIO PÚBLICO Y PRIVADO EN LA MODERNIDAD } \\
\hline ESPACIO PRIVADO FEMENINO & ESPACIO PÚBLICO MASCULINO \\
\hline $\begin{array}{l}\text { - Trabajo reproductivo } \\
\text { - Trabajo invisible } \\
\text { - Espacio identificado con la } \\
\text { naturaleza, el deseo, los } \\
\text { particularismos } \\
\text { - } \begin{array}{l}\text { Mujeres idénticas (madres y } \\
\text { esposas que cuidan) }\end{array}\end{array}$ & $\begin{array}{l}\text { - Trabajo productivo } \\
\text { - Salario familiar } \\
\text { - Espacio identificado con la cultura, } \\
\text { libertad, universalidad, la razón } \\
\text { - Espacio de hombres, individuos } \\
\text { iguales que producen }\end{array}$ \\
\hline
\end{tabular}

Fuente: elaboración propia

Para justificar esta asimétrica configuración ha sido clave el papel legitimador de los intelectuales, quienes o a lo largo de la historia han hecho dos cosas: legitimar o cuestionar el orden social. A menudo, han utilizado el mismo concepto de 'naturaleza' para ambas opciones. Así, Aristóteles justifica por naturaleza la desigualdad extrema como es la esclavitud y con el mismo concepto Locke afirma que todos los hombres nacen por naturaleza libres e iguales.

El Feminismo en sus distintas corrientes se caracterizará por desarrollar un corpus teórico que explica la realidad y no sólo analiza, sino que genera teoría política orientada hacia toda la sociedad, no sólo a las mujeres. Crea conceptos nuevos (uno de los más potentes es el de patriarcado), con los que interpela a todas las áreas de conocimiento para revisar sus fundamentos, sesgados por una visión androcéntrica. 
Entendemos por patriarcado el sistema social basado en el dominio de los hombres adultos sobre las mujeres y sobre los jóvenes de ambos sexos. Se ha utilizado más para investigar la desigualdad entre sexos, pero también ha sido utilizado, menos veces, para dar cuenta de otra desigualdad, la etaria. Los jóvenes son definidos no por lo que son sino por lo que pueden llegar a ser desde el adulto-centrismo.

Otro rasgo del feminismo es que tiene una agenda política vindicativa que arranca de la propia Ilustración y en su evolución histórica sigue teniéndola, y además se solidariza con los movimientos sociales que intentan una sociedad más libre y más justa (los derechos de los negros en EEUU, el medio ambiente, y todo lo que suponga más derechos para mujeres y hombres ciudadanos democráticos).

En una primera fase del Feminismo Ilustrado (1673-1791) destacan autores como Poullain de la Barre, filósofo feminista y cartesiano quien escribe en 1673 'De la Igualdad de los dos sexos' donde se muestra a favor de la igualdad entre mujeres y hombres. Fue discípulo de Rousseau al igual que Mary Wollstonecraft quien con su 'Vindicación de la Mujer' arranca el corpus teórico de la teoría política denominada Feminismo. Utiliza los mismos argumentos de su maestro Rousseau ya que no le parece razonable no considerar a la mujer como ciudadana, como sujeto político en equidad con el hombre.

Cuando se proclama la Declaración de Los Derechos del Hombre y del Ciudadano (1789), previa a la Primera Constitución Democrática (1791), Olympe de Gouges redactó otra que era una copia exacta para las mujeres Declaración de los Derechos de la Mujer:

Ésta debe ser la misma para todos: todas las ciudadanas y todos los ciudadanos, siendo iguales ante sus ojos, deben de ser igualmente admisibles a todas las dignidades, puestos y empleos públicos, según sus capacidades, y sin otras distinciones que aquellas de sus virtudes y sus talentos (Artículo 6).

Desde el mismo momento del surgimiento del feminismo aparece la reivindicación del derecho de las mujeres a la educación. Así lo expresa Mary Wallstonecraft (1792):

...para hacer al género humano más virtuoso y, por supuesto, más feliz, ambos sexos deben actuar desde los mismos principios...Para hacer realmente justo el Pacto social, y para extender los principios ilustrados...debe permitirse que las mujeres fundamenten la virtud en el conocimiento, lo que apenas es posible si no se las educa mediante las mismas actividades que a los hombres.

Es curiosa, la modernidad que emana de este escrito que data de 1792. Mary Wallstonecraft critica de su maestro que excluyera a las mujeres con relación a los derechos de los varones, como ser considerados individuos, ciudadanos con derecho a la educación; la argumentación se fundamenta de nuevo en el concepto de naturaleza. Rousseau sitúa a las mujeres en la naturaleza (excluidas del contrato social) y a los hombres en la cultura.

En efecto, hay una contradicción en Rousseau que llama la atención. El autor de El contrato social: o los principios del derecho político (1762), muestra sus ideas de forma muy radical 
en contra de la sociedad estamental. Defensor de la educación pública para los niños con el objetivo de aprender a ser un buen ciudadano. A este respecto, publicó El Emilio en el que sienta las bases de la meritocracia frente a los derechos de sangre y nacimiento, propios del Antiguo Régimen. Pero sólo incluía a los niños varones, pues todas las mujeres son excluidas, por el hecho de ser mujeres. Se da la paradoja de que el crítico más radical de la Ilustración no defienda para las mujeres el derecho a ser educadas y lo expresa en su libro Sofía en el que aconseja que desarrollen su naturaleza en el ámbito privado.

¿Por qué el autor más radical de la Ilustración niega a las mujeres la igualdad con los hombres, a pesar de haber inspirado a F. Babeuf -100 años antes que Marx- la 'Conspiración de los Iguales '(1796)? Precisamente esta conspiración tenía el objetivo de derrocar al Directorio e instaurar un régimen que garantizara la igualdad perfecta. Según Rousseau las mujeres están destinadas a obedecer, servir y cuidar a los hombres y en consecuencia no deben poseer criterio propio, pues de lo contrario no podrían realizar con éxito su misión derivada de la misma naturaleza. La educación para ellas se debería basar en un aprendizaje de mansedumbre que las convenciera de su incapacidad para discernir lo adecuado.

El concepto de naturaleza ha producido muchos estragos, pues lo natural no se puede cambiar. Sin embargo, las relaciones sociales de género se han ido construyendo a lo largo de la historia y precisamente porque no son naturales las podemos cambiar, y podríamos hacerlo mejor.

La segunda ola del feminismo dura todo un siglo (1848-1948) y pone en su agenda la vindicación del sufragio femenino. Se inicia con la consolidación de las revoluciones burguesas (1848) hasta 1948, con la Declaración Universal de Los Derechos Humanos, que fue posible gracias a los contactos y organización realizados por una mujer: Eleanor Roosevelt, viuda del presidente que había ganado la Segunda Guerra Mundial. Este documento marca un hito en la historia de los Derechos Humanos. Elaborada por representantes de todas las regiones del mundo con diferentes antecedentes jurídicos y culturales. Fue proclamada por la Asamblea General de las Naciones Unidas en París, el 10 de diciembre de 1948 como un ideal común para todos los pueblos y naciones. La Declaración establece, por primera vez, los derechos humanos fundamentales que deben protegerse en el mundo entero. Debemos a una mujer, Hansa Metha, la sustitución de la frase "todos los hombres nacen libres e iguales", por ésta: "todos los seres humanos nacen libres e iguales". Uno de los puntos es la igualdad entre mujeres y hombres tanto en el matrimonio como en su disolución:

Los hombres y las mujeres, a partir de la edad núbil, tienen derecho, sin restricción alguna por motivos de raza, nacionalidad o religión, a casarse y fundar una familia, $y$ disfrutarán de iguales derechos en cuanto al matrimonio, durante el matrimonio y en caso de disolución del matrimonio. (Artículo 16 de la Declaración Universal de los Derechos Humanos).

La tercera ola del Feminismo que podríamos datarla desde los años 90 hasta la actualidad, está impregnada de postmodernidad; amplía la agenda con la vindicación de derechos civiles y se transforma en un feminismo más global y complejo, ya que el propio feminismo se 
amplía con muchas corrientes influidas por la teoría Queer, el ecofeminismo o la reivindicación transexual, incluso un feminismo dialógico que se basa en la inclusión de otras mujeres pertenecientes a otras culturas y estratos sociales que no se sienten incluidas en el movimiento feminista occidental. Todo ello amplia la explicación de nuevas dimensiones de la desigualdad y su superposición. La agenda se diversifica, pero sigue estando en ella algo muy grave, como es la erradicación de todo tipo de violencia contra las mujeres, expresión extrema del patriarcado.

Es reseñable la paradoja que supone dos fenómenos en paralelo de signo contrario: por un lado, el gran éxito de la teoría feminista, desde el mismo momento de la entrada en la modernidad, y por otro, el desarrollo de un antifeminismo, que algunos lo convierten en un adjetivo peyorativo. Ello nos estaría explicando la gran inquietud que provoca los cambios de roles de género. En el fondo refleja la persistencia del patriarcado y de la misoginia.

En lo epistemológico, el feminismo interpela a todas las áreas científicas para que solucionen el sesgo producido durante la institucionalización de los saberes y las profesiones, ya que las mujeres no estaban. En la literatura socioeconómica los paradigmas clásicos centrales han estado alejados de las aportaciones procedentes de la teoría feminista, generalmente mujeres, madres fundadoras, que han sido silenciadas hasta hace relativamente poco tiempo. Y aún hoy muchas científicas feministas están en la frontera de sus áreas de conocimiento, muy alejadas del paradigma central de las mismas.

Si bien la ciencia aparece neutral, en su proceso de elaboración incorpora valores sociales, ya que en las ciencias sociales sujeto y objeto están en el mismo espacio. Las mujeres se sorprenden de que la ciencia sea ciega a sus demandas, por ello, E. Fox Keller (2001) se plantea que la investigación sobre género es más bien una reflexión sobre el uso y sentido, en ocasiones parcial y restrictivo, que han dado al conocimiento científico y que denominamos 'androcentrismo cientifico', es decir, la identificación de lo masculino con lo humano en general y, a su vez, equiparación de todo lo humano con lo masculino.

La teoría feminista en su afán de explicar la desigualdad entre mujeres y hombres sigue introduciendo nuevos conceptos. Uno de los más potentes es el señalado anteriormente (patriarcado) del que luego derivaría el de 'sistema de género' (Rubin,1996). La autora acuña este concepto en su artículo "El tráfico de mujeres: notas sobre la economía política del sexo". El sistema sexo/género lo define como el conjunto de disposiciones por el que una sociedad transforma la sexualidad biológica en productos de la actividad humana, y en el cual se satisfacen esas necesidades humanas transformadas. Rubin aborda el pensamiento marxista identificando el papel de las mujeres dentro de una sociedad capitalista. Considera que la reproducción de la fuerza de trabajo depende de las tareas domésticas de las mujeres para transformar las mercancías en sustento del trabajador. El sistema capitalista no puede generar excedentes sin las mujeres, sin embargo, la sociedad no les otorga a ellas ningún acceso al capital resultante.

Cuando hablamos de sistema de género-sexo aludimos por un lado a una categoría analítica crítica pero también a una categoría con un valor utópico, como señala Celia Amorós y Ana de Miguel (2005): 
El sistema de género-sexo como matriz que configura la identidad, así como la inserción en lo real de hombres y mujeres, es inseparable de su puesta en cuestión, como sistema normativo: sus mecanismos, como los de todo sistema de dominación, solamente se hacen visibles a la mirada crítica extrañada: la mirada conforme y no distanciada los percibe como lo obvio..., es decir, ni siquiera los percibe. (p. 16)

En Sociología el paradigma teórico imperante en buena parte del siglo XX fue el Funcionalismo, siendo Talcott Parsons quien argumentaba sobre la complementariedad de roles entre hombres y mujeres, teoría que fue muy criticada por las feminis tas de la época, como Betty Friedan y Kate Millet. En efecto, la combinación de categoría analítica y utopía política transformadora de toda la sociedad tiene como objetivo la satisfacción de las necesidades humanas (Frutos: 2008, 2013, 2016).

Ma Ángeles Durán (1996, 2019) considera un auténtico reto llevar a cabo una reinterpretación de la cultura teniendo en cuenta la aportación de la cultura femenina y su valoración a la par de la de los varones. Ambas culturas han contribuido a la evolución de la humanidad, aunque las mujeres han estado ausentes de los lugares de la producción de ciencia y conocimiento. La Sociología de género es una especialización que surge del debate entre la teoría feminista y la teoría sociológica. Ello ha permitido aplicar conceptos basados en un nuevo paradigma crítico, en el sentido que da Kuhn (1980) a las rupturas conceptuales, cuando un paradigma científico es sustituido por otro.

Así, a través del patriarcado diferentes corrientes feministas han podido explicar la distinta posición de mujeres y hombres en los espacios públicos/privados, productivo/reproductivo que justifican gran parte de las desigualdades existentes en la sociedad actual. Como señala Ma Jesús Izquierdo (2016, p.104):

La división sexual del trabajo es una característica de la organización de las actividades productivas, y es también un mecanismo básico de socialización.

El patriarcado se da en todos los niveles de la sociedad: político, económico e ideológico, a modo de estructura. La relación social de género es asimétrica y supone la dominación masculina sobre las mujeres en el conjunto de los espacios sociales (Bourdieu, 2006). Pierre Bourdieu, nacido en Argel, con su descripción etnográfica de la sociedad cabileña, auténtica reserva del inconsciente mediterráneo ofrece un instrumento extremadamente poderoso para explorar las estructuras simbólicas de ese inconsciente dominio masculino sobre las mujeres. El feminismo remarca que el orden social de género no es un orden natural contra el que nada pueda hacerse, sino que es un constructo- sociohistórico y, por ello, recordamos la famosa frase de Simonne de Beauvoir en el Segundo sexo 'la mujer no nace sino se hace'.

¿Por qué la desigualdad de género está tan oculta? Es anterior a la de clase social; es un tipo de desigualdad que ha pasado a ser invisible porque es la más antigua y se da en todas las sociedades en mayor o en menor grado. Recordemos el agradecimiento diario de Platón a los dioses por no haber nacido mujer. 
Alcañiz (2015, p. 47) comenta: “Todos estos comportamientos aluden a una distribución de roles en el que el poder y el control por parte de los hombres queda explicitado poniendo de manifiesto la continuidad en el estereotipo patriarcal del sistema de géneros".

Las mujeres, por el hecho de ser mujeres, se ven obligadas a cumplir un contrato no escrito, de carácter 'adscriptivo' de cuidados a las familias, de trabajo no remunerado, que se traduce a menudo en dobles jornadas y que influye en un posicionamiento más débil en el mercado de trabajo, en relación con los hombres.

Marión Young (1996) hace referencia a las caras de la opresión que se manifestarían en grupos sociales, expresando cinco formas:

1. No valorar económicamente el trabajo en sentido amplio de las ciencias sociales, como ocurre con el trabajo no remunerado realizado fundamentalmente por las mujeres o cuando los estudiantes realizan trabajos sin remuneración que son considerados como formación.

2. Excluir a determinadas poblaciones de la participación social como ocurre con el 'techo de cristal' sufrido por las mujeres en ciertos ámbitos profesionales a pesar de contar con la formación requerida o cuando los jóvenes son tachados con etiquetas despreciativas socialmente (los 'ninis').

3. Carencia de poder, como ocurre con la ausencia de representación en las organizaciones de población femenina y de jóvenes.

4. Imposición cultural de un grupo social sobre otro, como ocurre con la valoración masculina sobre la femenina y con la valoración del adulto sobre las personas jóvenes, o la cultura propia sobre la cultura del otro (extranjero).

5. Las expresiones de violencia en todos los ámbitos (física, social).

\section{Discusión: debate actual sobre los conceptos clase y género.}

Empecemos por el concepto de clase social que es relacional e histórico, aunque para algunos autores, este concepto estaba ya periclitado ante las nuevas realidades sociales capitalistas de mediados del siglo XX (Nisbet, 1959).

El concepto de clase se enmarca en la tradición marxiana frente al de estrato que tiene más que ver con la tradición funcionalista. Marx tenía una visión dicotómica de las clases, cuyo fundamento se basaba en la propiedad o no de los medios de producción. Como dijimos, el criterio que prevalece es el económico y no el jurídico que era la base de la sociedad estamental. En efecto, Marx distingue entre la burguesía, que es la clase dominante dueña de los medios de producción que se apropia de parte del trabajo realizado por el proletariado industrial, ya que el salario paga la fuerza de trabajo del obrero, pero no alcanza al pago total del trabajo. Ahí es donde se produce la explotación. La gran aportación de Marx es la teoría del valor. Las plusvalías que son los beneficios de los dueños de los medios de producción 
del capital industrial, al apropiarse de parte del trabajo realizado por los trabajadores. Esta concepción dicotómica la aplica también al materialismo histórico. Así, en las sociedades esclavistas antiguas la explotación se produce entre amo y esclavo y en las sociedades cristiano- feudales la explotación se da entre señor y siervo. Deja sin resolver en la teoría el problema de las clases medias, cuestión que es resuelto por los neo-marxistas.

E.O.Wright, americano afincado en la tradición marxista europea da cuenta de lo ocurrido en la evolución del capitalismo. Mantiene en su clasificación las dos clases de Marx y las amplía con el concepto de 'contradicciones de clase'. Además, también se inspira en la aportación de Roemer en su Teoría General de la Explotación donde remarca que siempre que alguien se aprovecha del trabajo de otro se da explotación. Así, logra resolver el problema teórico de las clases medias, pero no explica la posición de las clases que no están en el espacio público del mercado de trabajo remunerado, representado mayoritariamente por mujeres. Para Wright (1983, p. 86):

La producción doméstica es el estado final de la producción capitalista y la mujer la obrera no pagada- subordinada indirectamente al capital (...) la división sexual del trabajo no crea una división de los intereses de clase fundamentales entre los maridos y sus esposas.

Una de las desigualdades que más cuesta erradicar de nuestra sociedad es la de género. Fundamentalmente se aprende en el proceso de socialización por parte de los dos sexos, de tal forma que el resultado parece un proceso 'natural' como si se tratara de elementos, como la lluvia o el trueno, sobre los que no pudiéramos hacer nada, contribuyendo a su ocultación.

Si bien las diferencias biológicas explican, por ejemplo, quién da a luz los hijos, por sí solas no pueden explicar por qué las mujeres deben ser las encargadas y las únicas responsables de las tareas del cuidado de enfermos, niños, población dependiente, en general. Se trata de un contrato sexual previo al contrato social, de carácter adscriptivo.

El debate clase género viene de antiguo, incluso dentro del movimiento feminista. Para una corriente (Firestone, 1976), las mujeres constituyen una clase social y para otra no, si bien ambas consideran que todas las mujeres se ven afectadas por algún problema de marginación y exclusión. La corriente que sí considera a las mujeres una clase social es heredera de la tradición marxista. Entre los distintos autores hay un acuerdo notable con relación a la ubicación de mujeres en un mercado segregado sexualmente, donde ellas se concentran en las posiciones más precarias y peor pagadas (Crompton, 1994). También Caínzos (1996, p.140) reflexiona sobre la elaboración de un esquema de clases en la actualidad y destaca la desigualdad como la dimensión más relevante a tener en cuenta:

las desigualdades de clase tienen que ver tanto con las relaciones distributivas, de intercambio y funcionales que se dan en las esferas de la producción y el mercado, como en la propia definición de limitación de la frontera entre incluidos y excluidos de la participación en ellas.

En este sentido Victoria Sendón (2002, p.22) señala que: 
una de las características fundamentales de la dominación masculina es que ha utilizado las diferencias a favor de la desigualdad (...) la diferencia nada tiene que ver con la desigualdad. Esta ha sido una consecuencia perversa (...) Si lo entendiéramos bien, veríamos que las diferencias encierran una potencialidad extraordinaria. Sin diferencias no hay cambio ni pluralidad, todo sería homogéneo y estático.

La teoría feminista considera nuclear una epistemología en torno a la estructura social basada no sólo en las relaciones sociales asimétricas en el capitalismo -burguesía y proletariado-, sino que debe incluir los roles de género que posicionan a las mujeres en el trabajo no remunerado, invisibilizando la explotación a la que se ven sometidas, incluso dentro de su propia familia.

Todavía hoy la economía no reconoce el trabajo del 'care' ejercido mayoritariamente por mujeres (trabajo reproductivo), ya que la EPA, fuente de datos homogeneizada internacionalmente, sitúa a las mujeres que trabajan dentro del hogar como amas de casa, en la inactividad. Así, por utilizar un ejemplo real, el trabajo remunerado de una cocinera en un restaurante se considera trabajo por los economistas clásicos, pero si cocina para los pobres de su localidad una gran paella en la plaza del pueblo, los economistas ortodoxos no lo consideran trabajo. Ello constituye un agujero epistemológico para el área económica y un problema político.

La construcción social del mercado de trabajo otorga una gran centralidad al trabajo productivo protagonizado por los varones quienes perciben un salario por su fuerza de trabajo de carácter familiar. Las mujeres, por el contrario, no perciben ningún reconocimiento público de ciudadanía y supone para ellas una posición vicaria, de dominación/subordinación, que diría Weber, con relación a los hombres.

Por último, en cuanto al debate sobre clase género, algunos autores de la estratificación social consideran que las mujeres con el acceso a la educación y al trabajo remunerado se ubican en una clase propia, en función de la categoría profesional que ocupen, pero cuenta también el nivel educativo, y también la renta. En cualquier caso, tanto las mujeres que sólo están en casa como las ocupadas en el mercado de trabajo se ocupan en mayor o menor grado de las actividades del cuidado. Y si comparamos hombres y mujeres ocupados, a través de la Encuesta de Usos del Tiempo las mujeres trabajan el doble de tiempo que los hombres en el cuidado familiar. La buena noticia es la reducción horaria en función de la edad. Las mujeres más jóvenes dedican menos que las de más edad, pero no se ha conseguido la igualdad entre hombres y mujeres.

\section{El acceso a la educación de mujeres y hombres en España desde una mirada cualitativa "violeta"}

Como ya he mostrado, desde el feminismo ilustrado, desde el mismo momento de entrada en la Modernidad, se plantea el derecho a acceder a la educación en equidad con los varones. Sin embargo, costó mucho tiempo conseguirlo y además ese logro fue por etapas. Primero hubo que conseguir el derecho propiamente dicho a la educación (que ya se consiguió en 
occidente, pero no al mismo tiempo en cada país) y una vez conseguido, se demandó que chicos y chicas estudiaran lo mismo, que tuvieran el mismo curriculum vitae, cuestión que también se ha logrado en España, como en el resto de los países occidentales. Sin embargo, hay una tercera fase que aún no se ha llegado en ningún país y es que el rendimiento de los títulos educativos en el mercado de trabajo sea similar al que obtienen los hombres (Frutos: 2008, 2016).

España, apenas hace un siglo, tenía un nivel de analfabetismo muy alto especialmente femenino. En realidad, el Estado no asumía la obligatoriedad de formar a las niñas. A lo más, aconsejaba que si una niña quería aprender a leer y escribir la maestra podía ayudarla.

En cuanto a la segregación en las escuelas, si volvemos a la historia sabemos que en los tiempos que ha existido un distinto modelo genérico para chicos y chicas, las niñas han sido apartadas de la educación o, en el mejor de los casos, les ofrecían un tipo de escuela de muy bajo nivel de contenidos ya que la centralidad del curriculum era el aprendizaje de las labores de hogar, rezos y prácticas religiosas. Por ello, tras muchas luchas y con el empuje de sectores avanzados de final del s.XIX, que en el caso de España fue la Institución Libre de Enseñanza, se propone como avance y modernización de la sociedad las Escuelas Mixtas. A principios del siglo XX se difunde el argumento de que el camino más adecuado para lograr la escolarización de las niñas es la implantación de la Escuela Mixta con nuevos modelos pedagógicos. Esta idea es apoyada por las sufragistas de EE. UU. y de Europa. En el caso de España se extiende la idea durante la Segunda República, pero con ataques procedentes de los sectores más conservadores y de la Iglesia Católica, incluso algunas feministas no estaban de acuerdo con este tipo de escuela.

Hay dos momentos históricos muy relevantes a recordar en España:

$>$ Por ley, las mujeres no consiguieron el derecho al título universitario hasta 1912 (en época del rey Alfonso XIII).

$>$ El logro de la escolarización total de la población escolarizable de ambos sexos data de 1985.

Una primera conclusión es que el derecho a la educación se logra con perspectiva histórica, hace poco tiempo, es decir, muy tardíamente.

Para las niñas españolas fue muy importante las reformas educativas que implicaban obligatoriedad para cursar más años de estudio, fundamentalmente la Ley General de Educación de 1970 con la UCD (que amplia la obligatoriedad de los 10 a los 14 años), y la LOGSE elaborada bajo mandato del partido socialista que incrementa la escolarización obligatoria para ambos sexos, dos años más (hasta los 16 años). Estas decisiones beneficiaban especialmente a las niñas, sobre todo si procedían de familias con pocos recursos, pues en primer lugar si no había para todos los hijos, los padres decidían dar estudios a los hijos varones, ya que iban a ser los futuros cabezas de familia; era el modelo del "ganapán" y la estrategia familiar para las hijas era conseguir una buena boda (es decir un marido con buena posición). El riesgo era en caso de separación, porque las mujeres se empobrecían. Sin duda, el logro educativo va a influir en la autonomía de las mujeres tal y como pensaban Stuart 
Mill y su esposa Harriet Taylor, que podrían representar un feminismo liberal. En efecto, consideraban que las mujeres debían trabajar para no depender de sus maridos, idea que resulta muy actual.

En los países occidentales hemos asistido a un aumento de los niveles educativos de la población de ambos sexos; este incremento lleva un cambio social nuevo: los varones que tradicionalmente adquirían más educación que las mujeres se quedan atrás y son adelantados por primera vez en España, por sus compañeras. En efecto, se produce el 'sorpaso' y las mujeres adquieren niveles más altos de educación que sus compañeros varones. Ello quita argumentos a la explicación por parte de la Teoría del Capital Humano -que todavía influye mucho en instituciones nacionales e internacionales- del porqué los salarios femeninos son más bajos que el de sus compañeros. Su argumentación no explica esa realidad, pues sí que han invertido en educación.

El gran cambio se produce con el acceso educativo en los países del Estado de Bienestar europeos y sobre todo en los del sur de Europa porque partían de posiciones más bajas, especialmente en el caso de las mujeres. Este cambio social se intensifica en las tres últimas décadas del siglo XX como apreciamos en la siguiente tabla referida a la Educación Superior.

\begin{tabular}{|c|c|c|c|c|c|c|c|c|c|}
\hline EVOLUCIÓN D & $\overline{\mathbf{A T}} \mathrm{T}$ & BR & $\begin{array}{l}\text { A DE } \\
\text { E LA }\end{array}$ & TI & $\begin{array}{l}\text { CIÓ } \\
\text { CXO. }\end{array}$ & $\begin{array}{l}\text { EN LA } \\
70-71 /\end{array}$ & $\begin{array}{l}\text { UCA } \\
9-20\end{array}$ & ÓN S & ERIOR \\
\hline & & urso 7 & & & urso 9 & & & Irso 99 & 000 \\
\hline PAÍSES & Total & Mujer & Hombre & Total & Mujer & Hombre & Total & Mujer & Hombre \\
\hline SUECIA & 21,8 & 18,9 & 24,6 & 32,0 & 35,3 & 28,9 & 66,3 & 79,1 & 54,2 \\
\hline DINAMARCA & 18,9 & 14,2 & 23,2 & 36,5 & 39,0 & 34,1 & 56,1 & 65,2 & 47,4 \\
\hline FINLANDIA & 13,1 & 13,0 & 13,2 & 48,9 & 52,1 & 45,9 & 83,8 & 91,9 & 76,0 \\
\hline ITALIA & 16,7 & 12,8 & 20,5 & 32,1 & 31,1 & 33,1 & 46,6 & 52,8 & 40,7 \\
\hline BÉLGICA & 17,0 & 12,6 & 21,3 & 40,2 & 39,6 & 40,7 & 57,0 & 60,6 & 53,5 \\
\hline HOLANDA & 19,6 & 11,1 & 27,6 & 39,8 & 36,1 & 43,5 & 52,2 & 53,2 & 51,1 \\
\hline REINO UNIDO & 14,4 & 9,8 & 18,9 & 30,2 & 29,4 & 30,9 & 57,8 & 64,2 & 51,8 \\
\hline GRECIA & 13,1 & 8,5 & 17,4 & 36,1 & 35,8 & 36,3 & 54,7 & 56,2 & 53,2 \\
\hline IRLANDA & 11,9 & 8,3 & 15,3 & 29,3 & 27,7 & 30,8 & 46,4 & 51,4 & 41,6 \\
\hline ALEMANIA & 12,0 & 7,2 & 16,6 & 35,2 & 32,8 & 37,4 & 56,2 & 58,8 & 53,6 \\
\hline PORTUGAL & 6,6 & 5,8 & 7,3 & 23,2 & 26,1 & 20,3 & 47,1 & 53,9 & 40,4 \\
\hline ESPAÑA & 8,7 & 4,7 & 12,6 & 36,7 & 38,3 & 35,1 & 57,6 & 62,3 & 53,0 \\
\hline FRANCIA & 18,8 & - & - & 39,6 & 42,8 & 36,6 & 52,5 & 58,1 & 47,2 \\
\hline
\end{tabular}




\begin{tabular}{|ll|l|l|l|l|l|l|l|l|l|}
\hline $\begin{array}{l}\text { TOTAL } \\
\text { EUROPEA }\end{array}$ & U. & 14,8 & 10,6 & 18,2 & 35,4 & 35,9 & 34,9 & 62,4 & 62,1 & 51,1 \\
\hline
\end{tabular}

Fuente: Lola Frutos, a partir de la UNESCO, Institute for Statistics, 2002

La tendencia que observamos en la tabla es que en los últimos 30 años el alumnado se ha duplicado y en el caso de las mujeres se ha triplicado (Frutos, 2016). En el curso 1970-71, sólo 15 de cada 100 personas europeas accedían al nivel superior de estudios y veinte años después, en el curso 90-91 la tasa bruta de participación en la educación superior es del 35,4\%, y en el caso de España alcanza al 36,7\%. En el curso 99-2000 la tasa de participación es del 62,4\% y desde luego es espectacular el incremento en España, hasta el 57,6\%.

A pesar de la expansión de la Educación Superior en España, hace sólo 4 años que las mujeres obtienen el título de Doctor en un porcentaje mayor que los hombres.

\section{De la teoría a los datos. La investigación sobre la desigualdad educativa en niveles universitarios: segregación y rendimiento de los títulos. Resultados de investigación}

La desigualdad de oportunidades educativas (DOE) es un campo de investigación tradicional para los sociólogos. En la actualidad hay un debate no resuelto todavía acerca de si ha habido estabilidad o disminución de la DOE, a pesar de que se han desarrollado innovaciones metodológicas y teóricas tanto en el ámbito internacional (Golthorpe, 2007), como en España.

La tradición en España de este tipo de investigación viene de largo. Ciñéndome a la Educación Superior contamos con referencias importantes que ordenaré por el año de publicación. Se remonta a los estudios de Julio Carabaña (1985), quien basándose en estadísticas de enseñanza (INE) considera como variable independiente la clase social de los padres utilizando como indicador la categoría ocupacional.

Torres Mora (1994) realiza la misma investigación, pero con otra fuente (los Censos de población de 1970 y 1981). En otros estudios (Julio Carabaña 20004a,2004b, 2007) analiza el acceso a la universidad y la finalización de la educación superior, considerando variable independiente de origen social, la clase de los padres en lo ocupacional y el nivel educativo de los padres; utiliza datos transversales (100.000 casos de la Encuesta Sociodemográfica y 2.360 casos de la EPA).

Una cuestión que se investiga desde una perspectiva crítica es el rendimiento por sexo, ya que ellas estudian más, repiten menos y obtienen el título en una proporción superior a sus compañeros varones, sin embargo, a la hora de optar por determinadas carreras siguen el estereotipo de género, por lo que podemos hablar de 'carreras y sexo'. En efecto, las carreras que eligen las chicas son: ramas de la salud, ciencias humanas, sociales, pero donde no aparecen matriculadas es en las carreras técnicas que son las que más empleabilidad generan y donde se consiguen mayores salarios y reconocimiento. La investigación sobre esta 
cuestión aún no está clara. No estamos en condiciones de explicar por qué las chicas que obtienen mejores resultados académicos no optan más por carreras técnicas. En la siguiente tabla elaborada por el profesor José Saturnino (2021, p.29) observamos las opciones de ramas de conocimiento decididas por mujeres y hombres de distintas generaciones desde el curso 1956-1966 hasta el 2000-2001.

\begin{tabular}{|c|c|c|c|c|c|c|c|c|c|}
\hline \multicolumn{10}{|c|}{$\begin{array}{c}\text { Evolución de la tasa de varones y mujeres por ramas de conocimiento en la universidad y por } \\
\text { cohorte de nacimiento }\end{array}$} \\
\hline & \multicolumn{9}{|c|}{ COHORTES DE NACIMIENTO } \\
\hline & \multicolumn{3}{|c|}{ 1956-1966 } & \multicolumn{3}{|c|}{ 1977-1981 } & \multicolumn{3}{|c|}{$2000-2001 \cdot$} \\
\hline $\begin{array}{l}\mathbf{R} \\
\mathbf{A} \\
\mathbf{M} \\
\mathbf{A} \\
\mathbf{S}\end{array}$ & $\begin{array}{l}\text { Varones } \\
\%\end{array}$ & $\begin{array}{l}\begin{array}{l}\text { Mujer } \\
\text { es }\end{array} \\
\%\end{array}$ & $\begin{array}{c}\text { Total } \\
\%\end{array}$ & $\begin{array}{l}\text { Varones } \\
\%\end{array}$ & $\begin{array}{l}\text { Mujer } \\
\text { es } \\
\%\end{array}$ & $\begin{array}{l}\text { Varones } \\
\%\end{array}$ & $\begin{array}{l}\text { Varones } \\
\%\end{array}$ & $\begin{array}{l}\text { Mujeres } \\
\%\end{array}$ & $\begin{array}{l}\text { Varones } \\
\%\end{array}$ \\
\hline Total & 48,3 & 51,7 & 1.373 .312 & 41,9 & 58,1 & 1.405 .577 & 44,9 & 55,1 & 340.274 \\
\hline $\begin{array}{l}\text { Ingeniería } \\
\text { y } \\
\text { Tecnología }\end{array}$ & 87,0 & 13,5 & 195.010 & 79,3 & 20,4 & 179.914 & 76,7 & 23,3 & 58.249 \\
\hline $\begin{array}{l}\text { Ciencias } \\
\text { exactas y } \\
\text { naturales }\end{array}$ & 49,0 & 50,9 & 86.519 & 56,3 & 43,7 & 119.474 & 49,5 & 50,5 & 21.365 \\
\hline $\begin{array}{l}\text { CC. } \\
\text { Sociales y } \\
\text { Jurídicas }\end{array}$ & 50,1 & 49,9 & 427.100 & 36,2 & 63,8 & 701.383 & 40,2 & 59,8 & 164.429 \\
\hline $\begin{array}{l}\text { Humanida } \\
\text { des }\end{array}$ & 30,3 & 69,6 & 365.301 & 30,7 & 69,1 & 163.047 & 38,9 & 61,1 & 36.691 \\
\hline $\begin{array}{l}\text { CC. } \\
\text { Médicas y } \\
\text { Salud }\end{array}$ & 36,8 & 63,1 & 156.558 & 27,6 & 72,3 & 160.236 & 28,8 & 71,2 & 59.540 \\
\hline $\begin{array}{l}\text { Sin } \\
\text { especificar }\end{array}$ & 47,8 & 51,7 & 142.824 & 37,6 & 63,1 & 81.523 & - & - & - \\
\hline $\begin{array}{l}\text { Fuentes: } \\
\text { Micro } \\
\text { datos }\end{array}$ & \multicolumn{3}{|c|}{ ESD 1991 (INE) } & \multicolumn{3}{|c|}{$\frac{1}{\text { EPA } 1999 \text { (INE) }}$} & \multicolumn{3}{|c|}{ MINISTERIO DE EDUCACIÓN } \\
\hline
\end{tabular}

Fuente elaborada por J. Saturnino (2021) Es acceso a primer curso. El resto ya se tituló

En ella vemos que las opciones de las mujeres bajo la dictadura de Franco no impidieron que las mujeres siguieran sus estudios en la universidad, aunque exista una pequeña brecha con relación a los varones; las mujeres nacidas entre 1956 y 1966 eran mayoría en la institución universitaria, a pesar del contexto nacional-católico que exaltaba para las mujeres el rol de esposa y madre. La diferencia fundamental que quiero subrayar y coincido con el profesor José Saturnino es su escasa presencia en la rama de Tecnología e Ingeniería. Una de las razones que probablemente lleva a las mujeres universitarias a no optar por las carreras técnicas sea la situación en el mercado de trabajo, pues las empresas están pensadas para 
horarios masculinos y es allí donde las mujeres sienten el conflicto entre familia y trabajo, cuestión no resuelta desde la Transición Política (Frutos, 2012).

Entre 1990 y 2015 casi un tercio de los hombres y casi la mitad de las mujeres estudian en la universidad a los 19-20 años, pero no ha aumentado la igualdad de acceso a la institución. Lo más destacable es el aumento de los hijos de profesionales y la disminución de los hijos de agricultores, al tiempo que desde 1990 no se ha incrementado la proporción femenina tal y como se había dado con anterioridad.

Las señales del mercado de trabajo a los titulados se ven muy afectadas por la crisis económica. Los titulados universitarios se colocan más que los que tienen menos titulación. Pero los universitarios no se colocan como profesionales, sino como administrativos ganando menos. Sigue siendo interesante estudiar en la universidad, pero a causa de la crisis merece la pena más por el tipo de trabajo a conseguir que por el salario a ganar (Frutos, 2015, p.583)

Otra cuestión que se investiga es el rendimiento de los títulos en el Mercado de Trabajo desde el punto de vista feminista. En este caso mostraré resultados de investigación del Proyecto de I+D del que fui Investigadora Principal (Lola Frutos, 2003) ${ }^{2}$ Diferencias de género en el alumnado egresado de la Universidad y su inserción laboral (itinerarios académicos y laborales de mujeres y hombres). Estudio comparativo de la UMU, UNED y Santiago de Compostela. Además de la información ofrecida por las tres universidades, realizamos una Encuesta cuyos resultados más relevantes fueron los siguientes:

- Feminización de los títulos, pero los varones se gradúan en carreras con mayor empleabilidad (carreras técnicas). Ahora bien, las mujeres egresadas de esas titulaciones masculinizadas tienen más ventaja a la hora de obtener el primer empleo que sus compañeras de otras titulaciones.

- Se da ‘subempleo' o ‘sobre educación', si se prefiere, en el sentido de obtener trabajo en un puesto por debajo del nivel de formación adquirido en ambos sexos.

- Las egresadas están más representadas como funcionarias y los egresados como empresarios.

- La mayoría están ocupados como técnicos, pero conforme bajamos en el grado de autoridad jerárquica, la presencia femenina aumenta.

- En cuanto al salario, cuanto más elevado es hay menos mujeres.

Todas estas investigaciones otorgan una gran posibilidad de transformación desde la universidad a través de la investigación feminista utilizando fuentes debidamente desagregadas e indicadores que sirvan para visibilizar las desigualdades de género, ya que para actuar sobre la desigualdad hay que medirla.

Una primera cuestión es conseguir que las fuentes de datos estén desagregadas por sexo. A modo de ejemplo citaré los informes del PNUD que desde las últimas décadas del siglo

\footnotetext{
${ }^{2}$ Equipo de trabajo: Lola Frutos Balibrea (IP). Investigadores: Consuelo del Val Cid, Juan Carlos Solano. Colaboradores: Yolanda Agudo, Rita Radl Philipp.
} 
pasado publicaba sus indicadores, como el Índice de Desarrollo Humano (IDH) sin desagregar por sexo, hasta que en la IV Conferencia de Bejing de 1995 la presión feminista influyó para que conociéramos este indicador que incluye: renta, educación y esperanza de vida al nacer, desagregado por sexo. Considerando un mismo país perdía posiciones si se analizaban el IDH para mujeres y hombres que cuando se mostraba la posición sólo con ambos sexos.

Investigar con perspectiva crítica feminista precisa de teoría y de métodos que incluya nuevos indicadores de la desigualdad ya que, parafraseando a Kant "los datos sin teoría están ciegos, la teoría sin datos está vacía”.

\section{Referencias}

Alcañiz, M. (2015). Crisis, precariedad y desigualdad de género en España y en Italia. OBETS. Revista de Ciencias Sociales, 10, (1), 97-125.

Amorós, Celia y de Miguel, Ana (2005): Teoría feminista: de la Ilustración a la globalización. Madrid: Minerva Ediciones

Bourdieu, Pierre (2006). La dominación masculina, Madrid: Anagrama

Caínzos, M. (1996). Razones y recetas para construir un esquema de clases. REIS, $N^{o} 75$, pp. 109-143.

Carabaña, J. (1985): La igualdad de oportunidades ante la educación en España desde 1970, en Igualdad, desigualdad y equidad en España y Mexico, Madrid ICI-CM.

Carabaña, J. (2004a): Educación y movilidad social, en Sociología de la Educación, Barcelona, Barcanova.

Carabaña, J. (2004b): Una estimación del grado en que el número de hermanos deprime el nivel de estudios, en Reflexiones Sociológicas, Madrid, CIS.

Carabaña, J. (2007): Estado y Educación en la España Democrática, en Estado del Bienestar y competitividad: la experiencia europea, Madrid Fundación Carolina-siglo XXI.

Crompton, R. (1994). Clase y estratificación. Madrid: Tecnos.

De Gouges, Olympe (1792) (1993): La declaración de los Derechos de la Mujer y la Ciudadana. Valencia: Cátedra.

De la Barre, Poullain (1673). De la igualdad de los sexos. Texto completo en Dialnet. 
De la Boiétie, Étienne (1576 vo, 2019). Discurso de la servidumbre voluntaria, Madrid: Trotta.

Durán, M ${ }^{\mathrm{a}}$ Ángeles (1996). Mujeres y hombres en el futuro de la Ciencia. En Mujeres y Hombres en la Formación de la Teoría Sociológica, Madrid, CIS.

Durán, Mángeles (2019). Sobre ciencia, sabiduría y poder, Universidad de la Rioja (Discurso como Doctora Honoris Causa en la Universidad de la Rioja).

Eveling Fox, Keller (2001). Reflexiones sobre género y ciencia. Valencia: Alfons el Magnaním.

Firestone, Shulamith (1976). La dialéctica del sexo. Barcelona: Kairós.

Friedan, Betty (1965). La mística de la feminidad, Barcelona: Sagitario.

Frutos, Lola (2004). Educación superior e inserción laboral desde la perspectiva de género, en Reflexiones Sociológicas, Madrid, Centro de Investigaciones Sociológicas (CIS).

Frutos, Lola (2008). Situación Sociolaboral de mujeres y hombres en la Región de Murcia y en España: una relación asimétrica. Murcia, España: CES.

Frutos, Lola (2012). Trabajo y familia, ¿conciliación o conflicto? En J. García Marín, M. B. Gómez Vázquez (Eds.), Diálogos en la cultura de la paridad: reflexiones sobre feminismo, socialización y poder (pp. 163-192). Santiago de Compostela, España: Universidad de Santiago de Compostela.

Frutos, Lola (2016). Títulos, trabajos y profesiones, en Cristóbal Torres (ed.) España, 2015. Situación social, Madrid, Centro de Investigaciones Sociológicas (CIS).

Frutos, Lola (2016). Trayectorias académicas y profesionales de mujeres y hombres en España en un contexto de cambio. En Helena Xantana Cruz, Soraya Barreto Jannuario, María Eulina Pessoa de Carvalho (coords.), Entre Reflexòes e Practicas Feministas, pp.241-270, Brasil Série Estudos REDOR, UFPNE, CNPQ

Giddens, A. (1994). La estructura de clase en las sociedades avanzadas. Madrid, España: Alianza Editorial.

Izquierdo, María Jesús (2013). La construcción social del género, en C. Díaz y S. Dema (Eds.), Sociología y género (pp. 87-111). Madrid: Tecnos.

J. H. Goldthorpe, (2007). On Sociology: Numbers, Narratives, and the Integration of Research and Theory $\left(2^{a} e d\right)$. Oxford: Oxford University Press.

J. H. Goldthorpe, (2012). De vuelta a la clase y el estatus: por qué debe reivindicarse una perspectiva sociológica de la desigualdad social. REIS, 137, 43-58. 
Jackson Philip W. (1968). La vida en las aulas, Madrid: Morata.

Khun Thomas S. (1980). La estructura de las revoluciones científicas, Breviarios, Madrid: Fondo de Cultura Económica.

Millet Kate (1970). Sexual politics, New York, Doubleday \&Company. Traducido en español por la editorial Cátedra.

Nisbet, R. (1959). The decline and and fall of social class, Pacific Sociological Review, 2:1117.

Roemer John E. (1989). Teoría General de la Explotación y las Clases. Madrid: siglo XXI

Rubin Gayle (1996). El tráfico de mujeres: notas sobre la economía política del sexo. En Marta Lamas (Comp.), El género: la construcción cultural de la diferencia sexual. México: PUEGUNAM, 1996.

Saturnino Martínez García, José (2021). Género y educación: brecha inversa y segregación. En E. Ortiz et al.: Panorámica general de la equidad, educación y género en el siglo XXI, Murcia: Dirección General de la Mujer y Diversidad de Género.

Sendón, Victoria (2002). Marcar las diferencias: discursos feministas ante un nuevo siglo, Barcelona: Icaria.

Subirats, Marina, Brullet Cristina (1989). Rosa y azul. La transmisión de los Géneros en la Escuela Mixta. Madrid: Instituto de la Mujer.

Torres Mora, J.A. (1994): Las desigualdades en el acceso a la educación en España: un estudio socio-gráfico, [Tesis Doctoral, UCM]

Wollstonecraft, Mary (1791). Vindicación de los derechos de la mujer. Recuperado de https://cutt.ly/ebxSP9g.

Wright, E. O. (1983). Clase, crisis y Estado. Madrid, España: Siglo XXI.

Young, Marion (1996). Vías políticas y diferencia de grupos: una crítica del ideal de ciudadanía universal. En C. Castell (Coord.) Perspectivas feministas en teorías políticas, pp.99-127, Barcelona: Paidós. 